\title{
DEEPBLUR: BLIND IDENTIFICATION OF SPACE VARIANT PSF
}

\author{
Valentin Debarnot ${ }^{\dagger}$ \& Pierre Weiss ${ }^{\ddagger}$ \\ †Institut de Mathématiques de Toulouse, Toulouse, France, \\ ${ }^{\ddagger}$ Institut de Mathématiques de Toulouse \& CNRS, Toulouse, France.
}

\begin{abstract}
We propose to train a neural network to estimate space varying blur operators from a single blurry image. The key assumption is that the operator lives in a subset of a known subspace, which is a reasonable assumption in many microscopes. We detail a specific sampling procedure of the subset to train a Resnet architecture. This allows a fast estimation. We finally illustrate the performance of the network on deblurring problems.
\end{abstract}

Index Terms - blur identification, neural network, nonuniform blur, blind debluring, blind inverse problem.

\section{INTRODUCTION}

Optical systems often produce observations blurred ${ }^{1}$ by the various optical components. Most existing deblurring algorithms require the knowledge of the blur operator to improve the image quality. This assumption is critical to produce accurate reconstructions. There are three main methods for estimating a blur operator: i) using a theoretical model, which is prone to calibration errors, ii) imaging point sources: unfortunately, this step should often be reproduced before each acquisition, iii) solving a blind inverse problem, i.e. looking for both the original signal and the blur operator. In this paper, we are interested in the latter approach.

\subsection{Existing works}

Blind inverse problems have received continual attention for the last four decades. The approach that has prevailed is to estimate the original signal and the blur operator using an alternating minimization procedure. Under regularity assumptions on the image and the operator, each sub-problem boils down to a standard inverse problem [1, 2, 3, 4]. In the last few years, machine learning approaches have begun to emerge and outperform older methods, in particular in the field of computer vision where motion blurs are estimated [5, 6]. These blurs

This work was supported by the Fondation pour la Recherche Médicale (FRM grant number ECO20170637521 to V.D.). P.W. acknowledges support from the ANR JCJC OMS and from ANR 3IA ANITI.

${ }^{1}$ In this paper, we deal with spatially varying blurs, and therefore prefer the term deblurring to deconvolution (i.e. spatially invariant operator).

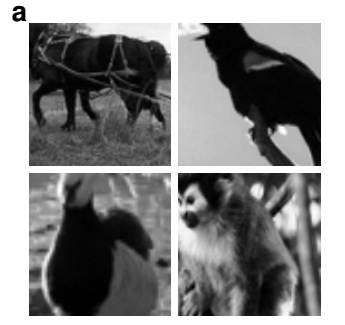

Image dataset

b
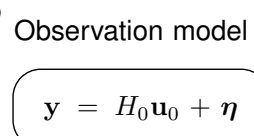

$H_{0} \sim \mathcal{L}_{H}$

$\mathbf{u}_{0} \sim \mathcal{L}_{u}$

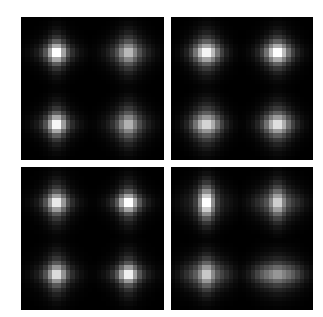

Operator dataset

c

Training procedure

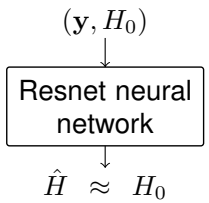

Fig. 1: a, Image and operator dataset used to train the proposed neural network. The image dataset is composed of 10000 natural images, and the operator dataset is composed of space varying blur operator. b, Observation model, the unknown blur operator $H_{0}$ and the unknown signal $u_{0}$ are drawn from an arbitrary distribution. $\mathbf{c}$, Estimation procedure, the blurry-noisy image is given to the Resnet neural network. It outputs $\hat{H}$ an estimate of the original blur operator $H_{0}$.

have characteristic (slow Fourier decay) which are not directly applicable to microscopic blurs. Recently [7] proposed to use a neural network to estimate the blur parameter from a single blurry image. This work is closely related to our contribution, with the following differences: i) their operators are convolutions and ii) a central assumption in our work is that the operator live in a low-dimensional subspace.

\subsection{Observation model}

Let $\mathbf{y} \in \mathbb{R}^{m}$ denote the observed signal, $H_{0}: \mathbb{R}^{m} \rightarrow \mathbb{R}^{m}$ denote the linear blur operator and $\mathbf{u}_{0} \in \mathbb{R}^{m}$ denote the original image. We have

$$
\mathbf{y}=H_{0}\left(\mathbf{u}_{0}\right)+\boldsymbol{\eta},
$$

where $\boldsymbol{\eta} \in \mathbb{R}^{m}$ is an additive random perturbation.

Without further assumption, estimating $H_{0}$ and $\mathbf{u}_{0}$ from $\mathbf{y}$ 
is an ill-posed problem. In the next paragraphs, we will detail the structure of the admissible operators.

\subsubsection{Product-convolution expansion}

Optical blurs are very often modeled by a convolution operator, and can only capture stationary degradations. We overcome this limitation by using the product-convolution expansions [8, 9]. They are efficient models of space varying blurs, have good approximation properties if the PSF (Point Spread Function) varies smoothly, and can be computed rapidly using fast Fourier transforms.

A product-convolution expansion $H$ has the following form:

$$
H(\mathbf{u})=\sum_{k=1}^{K} \mathbf{e}_{k} *\left(\mathbf{f}_{k} \odot \mathbf{u}\right), \forall \mathbf{u} \in \mathbb{R}^{m},
$$

where $*$ denotes the convolution product, $\odot$ denote the Hadamard (element-wise) product and $\left(\mathbf{e}_{k}\right)_{k}$ and $\left(\mathbf{f}_{k}\right)_{k}$ denote two families of $\mathbb{R}^{m}$. The family $\left(\mathbf{e}_{k}\right)$ can be interpreted as a basis to describe the PSFs of $H$ while the family $\left(\mathbf{f}_{k}\right)$ describes the PSF variations.

To clarify the definition, we provide an example with $K=$ 2 in Fig. 2. The family $\left(\mathbf{e}_{k}\right)$ is computed by selecting 2 anisotropic Gaussian kernels with standard deviations randomly chosen in $[1,4]$, see Fig. 2a The family $\left(\mathbf{f}_{k}\right)$ is computed with the following procedure:

1. Generate a smooth Gaussian process $v \in \mathbb{R}^{m}$.

2. Extract 2 random domains $\omega_{1}, \omega_{2}$ of $\mathbb{R}^{2}$ by thresholding $v$.

3. Construct 2 images $v_{1}, v_{2}$ as the indicator functions of $\omega_{1}, \omega_{2}$.

4. Return a smoothed version of these 2 images using a Gaussian kernel with large variance.

This allows to produce smooth and contrasted coefficients maps $\mathbf{f}_{k}$. Finally, we apply (2) to a Dirac comb in fig 2c.

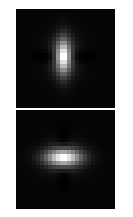

(a)

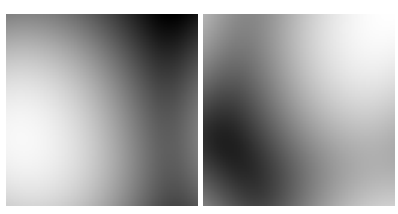

(b)

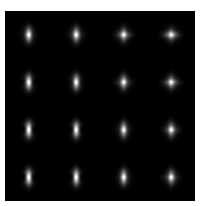

(c)
Fig. 2: Example of product-convolution. a) $24 \times 24$ anisotropic Gaussian filters $\mathbf{e}_{1}, \mathbf{e}_{2}$, b) $128 \times 128$ coefficients maps $\mathbf{f}_{1}, \mathbf{f}_{2}$, c) Product-convolution operator applied to a Dirac comb $(128 \times 128)$.

\subsubsection{Subspace of operators}

We now detail the assumptions on the space of admissible blur operators.
Assumption 1.1 (Subspace of operators). Let $\left(\mathbf{e}_{k}\right)$ and $\left(\mathbf{f}_{k}\right)$ denote two known families. The blur operators live in a lowdimensional subspace $\mathcal{H} \subset\left\{H: \mathbb{R}^{m} \rightarrow \mathbb{R}^{m}\right\}$ of productconvolution operators of the form:

$$
H[\gamma](\mathbf{u})=\sum_{k=1}^{K} \gamma_{k} \mathbf{e}_{k} *\left(\mathbf{f}_{k} \odot \mathbf{u}\right)
$$

We will refer to an operator in $\mathcal{H}$ either by $H, \gamma$ or $H[\gamma]$.

Assumption 1.1 is realistic in many microscopes. The space $\mathcal{H}$ can be estimated by imaging fluorescent micro-beads [10], or provided that we have access to a collection of operators [11].

Assumption 1.2 (A conical hull in $\mathcal{H}$ ). We are given a collection $\left(\gamma_{p}\right)$ of $P$ admissible operator parameterizations. Assuming that this set represents the set of operators sufficiently densely, we can construct the conical hull of the coefficients $\gamma_{p}$ :

$$
\mathcal{C} \stackrel{\text { def. }}{=} \text { cone }\left(\gamma_{p}, 1 \leq p \leq P\right),
$$

which we will use as a proxy to describe the set of all admissible operators.

\subsection{Contributions}

The objective of this paper is to recover an estimate $\hat{H}$ of $H_{0}$ and an estimate $\hat{\mathbf{u}}$ of $\mathbf{u}_{0}$ given the observation $\mathbf{y}$. Given the previous assumptions, a natural approach to attack this issue would consist in solving

$$
\inf _{\substack{\mathbf{u} \in \mathbb{R}^{m}, H \in H[\mathcal{C}]}} \frac{1}{2}\|H \mathbf{u}-\mathbf{y}\|_{2}^{2}+R(\mathbf{u}),
$$

where $R: \mathbb{R}^{m} \rightarrow \mathbb{R}_{+}$is a regularization function promoting desired properties of the signal $\mathbf{u}$. Imposing $H \in H[\mathcal{C}]$ is a strong regularization on the admissible operators that preserve properties of the admissible operators (e.g. positivity or barycenter). Optimizing $H$ and $\mathbf{u}$ jointly makes the problem (5) nonconvex and numerically involved.

In this paper, we therefore follow a different route and consider a two-step procedure: First, estimate the blur operator

Find $\hat{H} \in \mathcal{C}$ an estimate of $H_{0}$ using $\mathbf{y}$.

Second, solve a non-blind inverse problem assuming that $H_{0}=\hat{H}$.

The main contribution of this paper is to provide a robust methodology to solve the first step. We identify the blur operator from a blurry-noisy image by training a neural network. This requires a specific sampling procedure of the set $H[\mathcal{C}]$. We then train a Resnet neural network to estimate a spatially variant blur operator from a single blurry image. This procedure appears to be robust if trained with a large enough dataset. In particular, we show that the network trained with natural images still performs well on unseen biological images. A summary of the approach is shown in Fig. 1 


\section{METHOD}

\subsection{Modeling and sampling blur operators}

In order to train a neural network, we will need to sample operators at random within $H[\mathcal{C}]$. If $P$ is large, a uniform sampling on the $(P-1)$-dimensional simplex would result in the fact that the extreme rays of the cone $\mathcal{C}$ are not explored. To avoid this flaw, we propose two complementary solutions:

i) Simplify the cone by keeping only a subset of extreme rays.

ii) Do not draw the coefficients $\alpha$ uniformly, but rather favor sparse distributions.

\subsubsection{Cone simplification}

Simplifying a conical hull is a difficult problem related to nonnegative matrix factorization. We refer the interested reader to [12] for instance. In this work, we propose a simple greedy algorithm described in Algorithm 1

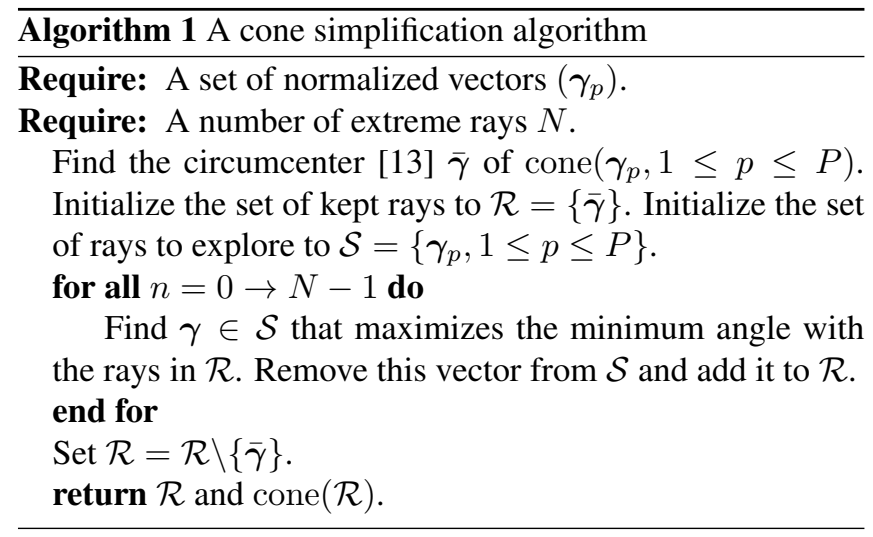

\subsubsection{Sampling the operators at random}

By Caratheodory theorem, any point within a conical hull can be expressed as a conical combination of $K$ extreme rays. In this work, we propose a heuristic method to favor the extreme rays. This is detailed in Algorithm 2

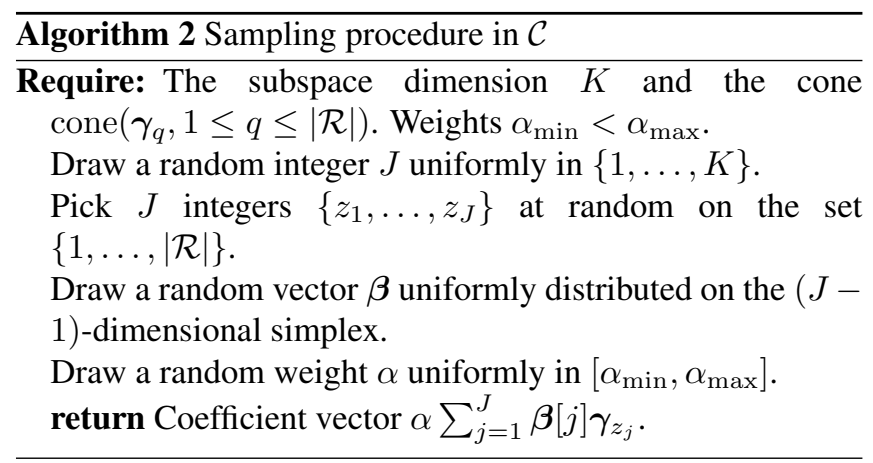

\subsection{Learning procedure}

The coefficient $\gamma$ is drawn from a distribution $\mathcal{L}_{H}$ defined in Algorithm 2 This ensures that $\gamma \in \mathcal{C}$. In order to compute $\mathbf{y}$ from the model (1), we need an image $\mathbf{u}$. In this work, we select $\mathbf{u}$ using the uniform distribution $\mathcal{L}_{\mathbf{u}}$ over the STL-10 data-set of natural images [14]. This data-set is composed of 10000 images for the training procedure, and 1000 different images for the testing procedure.

Finally, we train a Resnet convolutional neural network (CNN) [15] to retrieve $\gamma$ using the blurry-noisy observation y. We use the relative Frobenius norm between the true operator and the estimation as a cost function. We train the convolutional neural network with 100 iterations of the $A D A M$ algorithm, using 2,048 images drawn from the distribution $\mathcal{L}_{\mathbf{u}}$, with a batch size of 64 and a learning rate equal to $10^{-3}$.

\section{NUMERICAL ILLUSTRATION}

In this section, we work with square images $m=128 \times 128$. The subspace $\mathcal{H}$ and the convex set $\mathcal{C}$ are computed using the estimation procedure described in [10]. We use the operators described in Section 1.2.1. The resulting subspace is composed of $K=20$ elementary operators. The learning procedure is performed using the parameters described in Section 2.2 The average relative Frobenius norm between the true operator and the estimation produced by the network is less than $5 \%$ at the end of the training phase (on the test data-set).

To illustrate the proposed methodology, we solve the following total variation deblurring problem with a proximal gradient descent algorithm:

$$
\inf _{\mathbf{u} \in \mathbb{R}^{m}} T V(\mathbf{u})+\frac{\lambda}{2}\|\hat{H} \mathbf{u}-\mathbf{y}\|_{2}^{2} .
$$

In Fig. 3. we display the true and estimated operators applied to a Dirac comb, and the result of the deblurring algorithm using the estimated operator with total variation regularization for various images. In Fig. $3 \mathrm{a}-\mathrm{d}$, we use natural images of the STL-10 test data-set. As expected, the network - that has been trained on similar images - outputs an accurate estimate of the true operator. Solving the deblurring Problem (7) leads to sharp results, and allows to recover hidden details.

In Fig. 3e and Fig. 3f. we observe how the trained network behaves on other images. In Fig. 3e, we use a real image of microscopy from the dataset [16]. In Fig. 3f] we use a simulated image of single molecule localization microscopy, i.e. points sources at random locations. In both problems the network performs well and retrieves the operator with high accuracy. More importantly, solving the deblurring Problem (7) with the estimated operator allows to better discriminate the biological elements, such as the cells in Fig. 3e. In the last experiment, we replace the total variation regularization term by a $\ell_{1}$ penalty on the signal. It allows to retrieve sparse elements and greatly improves the resolution of fluorescent proteins. Notice that these deblurring results are simply a proof 
of concept since better deblurring algorithms could be used (e.g. a variational network).
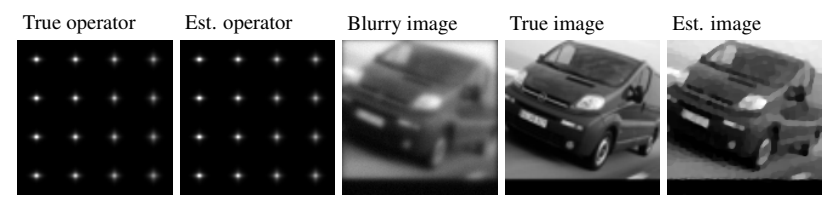

(a) Operator relative error: $4.8 \%$. SNR: $15 \mathrm{~dB}$ to $21 \mathrm{~dB}$.
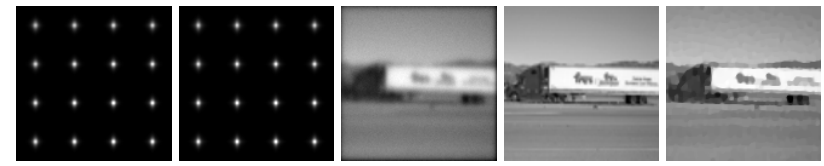

(b) Operator relative error: $3.4 \%$. SNR: $19 \mathrm{~dB}$ to $27 \mathrm{~dB}$.
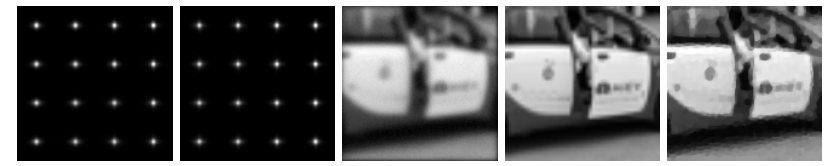

(c) Operator relative error: $6.8 \%$. SNR: $18 \mathrm{~dB}$ to $24 \mathrm{~dB}$.
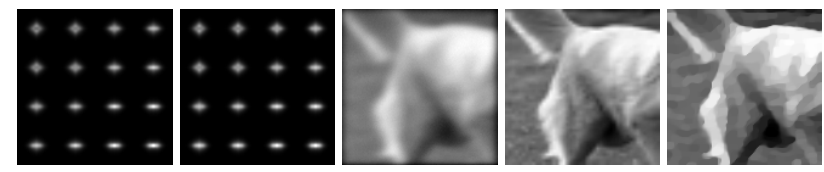

(d) Operator relative error: $4.9 \%$. SNR: $19 \mathrm{~dB}$ to $28 \mathrm{~dB}$.
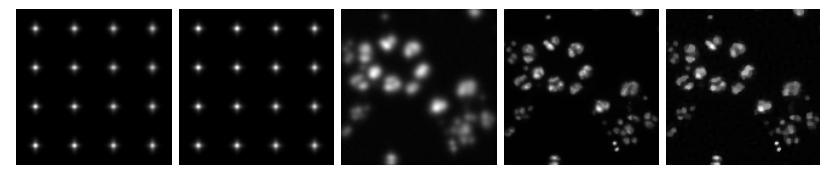

(e) Operator relative error: $6.5 \%$. SNR: $7.6 \mathrm{~dB}$ to $15 \mathrm{~dB}$.
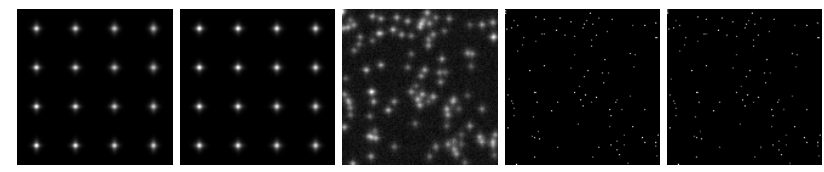

(f) Operator relative error: $8.4 \%$. SNR: $-7 \mathrm{~dB}$ to $0.64 \mathrm{~dB}$.

Fig. 3: From left to right. True and estimated operator, blurry-noisy image, original image and estimated one by solving problem (7) with total variation regularization for (a)-(e) and $\ell_{1}$ regularization for (f)). The SNR with the blurry and the estimated images is given in the sub-captions.

\section{CONCLUSION}

We have presented a learning based method to estimate spatially varying blur from a blurry-noisy image. This methodology is robust in the sense that even if the neural network is trained on a set of natural images, it still performs well on biological images not seen during the training. We will extend this work to blur operators adapted to large images estimated from microscopes in [10]. We expect this approach to provide a turnkey tool for biologists to improve their image quality.

\section{COMPLIANCE WITH ETHICAL STANDARDS}

This is a numerical simulation study for which no ethical approval was required.

\section{REFERENCES}

[1] Rob Fergus, Barun Singh, Aaron Hertzmann, Sam T Roweis, and William T Freeman, "Removing camera shake from a single photograph," in ACM SIGGRAPH 2006 Papers, pp. 787-794. 2006.

[2] Anat Levin, Yair Weiss, Fredo Durand, and William T Freeman, "Understanding and evaluating blind deconvolution algorithms," in 2009 IEEE Conference on Computer Vision and Pattern Recognition. IEEE, 2009, pp. 1964-1971.

[3] Libin Sun, Sunghyun Cho, Jue Wang, and James Hays, "Edge-based blur kernel estimation using patch priors," in IEEE International Conference on Computational Photography (ICCP). IEEE, 2013, pp. 1-8.

[4] Daniele Perrone and Paolo Favaro, "Total variation blind deconvolution: The devil is in the details," in Proceedings of the IEEE Conference on Computer Vision and Pattern Recognition, 2014, pp. 2909-2916.

[5] Jian Sun, Wenfei Cao, Zongben Xu, and Jean Ponce, "Learning a convolutional neural network for non-uniform motion blur removal," in Proceedings of the IEEE Conference on Computer Vision and Pattern Recognition, 2015, pp. 769-777.

[6] Dong Gong, Jie Yang, Lingqiao Liu, Yanning Zhang, Ian Reid, Chunhua Shen, Anton Van Den Hengel, and Qinfeng Shi, "From motion blur to motion flow: a deep learning solution for removing heterogeneous motion blur," in Proceedings of the IEEE Conference on Computer Vision and Pattern Recognition, 2017, pp. 2319-2328.

[7] Adrian Shajkofci and Michael Liebling, "Spatially-variant cnn-based point spread function estimation for blind deconvolution and depth estimation in optical microscopy," IEEE Transactions on Image Processing, vol. 29, pp. 5848-5861, 2020.

[8] Loïc Denis, Eric Thiébaut, Ferréol Soulez, Jean-Marie Becker, and Rahul Mourya, "Fast approximations of shift-variant blur," International Journal of Computer Vision, vol. 115, no. 3, pp. 253-278, 2015.

[9] Paul Escande and Pierre Weiss, "Approximation of integral operators using product-convolution expansions," Journal of Mathematical Imaging and Vision, vol. 58, no. 3, pp. 333-348, 2017.

[10] Valentin Debarnot, Paul Escande, Thomas Mangeat, and Pierre Weiss, "Learning low-dimensional models of microscopes," IEEE Transactions on Computational Imaging, 2020.

[11] Valentin Debarnot, Paul Escande, and Pierre Weiss, "A scalable estimator of sets of integral operators," Inverse Problems, 2019.

[12] Greg Van Buskirk, Benjamin Raichel, and Nicholas Ruozzi, "Sparse approximate conic hulls," in Advances in Neural Information Processing Systems, 2017, pp. 2534-2544.

[13] René Henrion and Alberto Seeger, "Inradius and circumradius of various convex cones arising in applications," Set-Valued and Variational Analysis, vol. 18, no. 3-4, pp. 483-511, 2010.

[14] Adam Coates, Andrew Ng, and Honglak Lee, "An analysis of singlelayer networks in unsupervised feature learning," in Proceedings of the fourteenth international conference on artificial intelligence and statistics, 2011, pp. 215-223.

[15] Kaiming He, Xiangyu Zhang, Shaoqing Ren, and Jian Sun, "Deep residual learning for image recognition," in Proceedings of the IEEE conference on computer vision and pattern recognition, 2016, pp. 770778.

[16] Vebjorn Ljosa, Katherine L Sokolnicki, and Anne E Carpenter, "Annotated high-throughput microscopy image sets for validation.," Nature methods, vol. 9, no. 7, pp. 637-637, 2012. 